\title{
Psychometric Performance of Resilience Scale for Chinese Adolescent (RSCA) for Undergraduates in Guangdong, China
}

\author{
American Journal of Education and \\ Learning \\ Vol. 6, No. 2, 86-93, 2021 \\ e-ISSN:2518-6647 \\ check for
updates
}

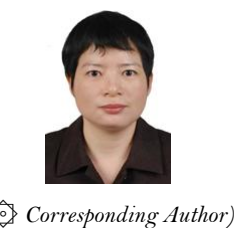

\author{
iD Hou Yongmei ${ }^{10}$ \\ iD Wang Yiyang ${ }^{2}$
}

Department of Psychology, School of Humanities and Administration, Guangdong Medical University, Dongguan, Guangdong Province, China.

Email:2184456621@qq.com

To analyze the psychometric performance of Resilience Scale for Chinese Adolescent (RSCA) for undergraduates in Guangdong. Stratified random sampling method was used to select 1628 undergraduates from 7 colleges in Guangdong. RSCA and Academic Burnout Scale for Chinese Undergraduates (ABSCU) were used to investigate them. Cronbach's $\alpha$ coefficient and split-half reliability were used to analyze the internal consistency of RSCA. Convergent validity, discriminant validity, factor analysis and criterion validity were used to evaluate its validity. Celling and floor effect were used to analyze its sensitivity. Cronbach's $\alpha$ coefficient of the total questionniar, 2 domains and 5 factors were0.72-0.86, which met the requirements of the group comparison. Spearman-Brown split-half coefficient of the total questionniar, 2 domains and 5 factors were $0.71-0.89$. The calibration success rate of convergent and discriminant validity of 5 factors were all $100 \%$. The calibration success rate of convergent and discriminant validity of 2 domains were all above $86.7 \%$. Five principal components obtained from 27 items, witha cumulative variance contribution rate of $48.79 \%$ and two principal components obtained from 5 factors, withacumulative variance contribution rateof $65.23 \%$, which basically metthe theoretical conception of RSCA. The total score of RSCA significantly predicted the total score of ABSCU $\left(R_{\mathrm{adj}}{ }^{2}=0.158, P<0.001\right)$. The total score, scores of 2 domains and 5 factors of RSCA were all normal distribution, without any celling or floor effect. The psychometric performance of RSCA for undergraduates in Guangdong were valid and reliable.

Keywords: RSCA, Resilience, Validity, Reliability, Sensitivity, Undergraduates.

DOI: $10.20448 / 804.6 .2 .86 .93$

Citation | Hou Yongmei; Wang Yiyang (2021). Psychometric Performance of Resilience Scale for Chinese Adolescent (RSCA) for Undergraduates in Guangdong, China. American Journal of Education and Learning, 6(2): 86-93.

Copyright: This work is licensed under a Creative Commons Attribution 3.0 License

Funding: This study received no specific financial support.

Competing Interests: The authors declare that they have no competing interests.

History: Received: 10 August 2021/ Revised: 13 September 2021/ Accepted: 5 October 2021/ Published: 28 October 2021

Publisher: Online Science Publishing 


\section{Highlights of this paper}

- With a large sample and multi center empirical research, this study analyzed the psychometric performance of Resilience Scale for Chinese Adolescent (RSCA) for undergraduates in Guangdong, which can fill the shortage of the standardization of the localization evaluation tool, further clarify the connotation of the concept of adolescent resilience, and provide useful reference for the evaluation and cultivation of teenagers' psychological quality.

\section{INTRODUCTION}

Resilience is a good ability to adapt to adversity, trauma and other major stress events. It is a process in which an individual can quickly recover and successfully cope with great pressure or danger through the dynamic interaction of a series of abilities and qualities (American Psychology Association, 2011). Resilience can change individuals' cognition of the risk of negative pressure and traumatic events, extract the favorable resources of pressure and events, so as to reduce the damage results, and improve individuals' physiological function and coping ability (American Psychology Association, 2011). Empirical research shows that people with high resilience have stronger stress tolerance (Chen, Li, \& Yang, 2020) and more stable emotions (Chen et al., 2020), so they can effectively safeguard self-esteem (Liu \& Zhouyuqiu, 2019), personal legitimate rights and interests (Li, Niu, \& Ma, 2020), better adapt to the environment (Cao, 2020), promote academic (Chen, Zhao, \& Wang, 2020; Sang, Chen, \& Zhu, 2016) and career performance (Gao \& Sui, 2018), have higher life satisfaction (Cao, 2020), stronger physical rehabilitation ability (Chen, Li, \& Yang, 2019), and are less prone to suffer from mental illness (Liu \& Zhouyuqiu, 2019) or suicidal ideation (Xu \& Yang, 2019). Due to the importance of resilience to individual development, it has attracted more and more attention from all walks of life. Research on resilience has been carried out earlier abroad, and many assessment tools have been developed, which are widely used in healthy people of all ages (children, adolescents, adults and the elderly) and clinical patients with different diseases (Bulut, Doğan, \& Altundağ, 2013; Lin, Luo, \& Wang, 2019; Pan \& Yang, 2013; Saide, 2019). However, Western measurement tools fail to cover the important content of Chinese resilience and the unique way of Chinese behavior (Heppner et al., 2006). Therefore, it is necessary to develop a localized measurement tool for Chinese resilience. Resilience Scale for Chinese Adolescent (RSCA) (Hu \& Gan, 2008) is a local scale compiled by Hu and Gan (2008). It has simple items, clear structure, and its content is suitable for the living conditions of Chinese adolescents. It is also committed to exploring a positive construct conducive to the healthy growth of adolescents. Therefore, since its establishment, it has been more and more widely used and recognized. But what is the psychometric performance of RSCA applied to college students? At present, there is a lack of relevant evidence. Based on the above analysis, this study intends to explore the psychometric performance of RSCA applied to college students in Guangdong Province by using a large sample and multi center survey model.

\section{OBJECTS AND METHODS}

\subsection{Objects \\ 2.1.1. Sample Size Estimation}

The minimum sample size is calculated by $\mathrm{G}^{*}$ power 3 (Cohen, 1992). Because resilience is a continuous variable, there is no difference between occurrence and non occurrence, so we can't calculate the incidence rate, and we can't directly use resilience to calculate the sample size. RSCA is used to evaluate mental health status, and academic burnout is one of the important indicators of health status, so we use the prevalence rate of academic burnout of college students to calculate the sample size. Previous studies have shown that the the detection rate of academic burnout among domestic college students is 24.8-51.3\% (Hou, Hu, \& Wang, 2017; Li, Yin, \& Liu, 2014; 
Yu, Yin, Zhao, \& Xin, 2020), the test effect value is medium level (Cao, 2020; Li et al., 2020; Liu \& Zhouyuqiu, 2019), that is, the $\mathrm{d}$ value is $0.50-0.80$ (Zhang \& Xu, 2012). In this study, we set the effect value $\mathrm{d}=0.70$, the statistical test power of $1-\beta=0.80$, the type I error probability $\alpha=0.05$, and the minimum sample size is calculated as 634 . The minimum sample size is determined as 761 due to a $20 \%$ of possible follow-up loss rate.

\subsubsection{Sampling}

Stratified random sampling method is used to selecte undergraduates from 7 universities including Sun Yat Sen University, Guangdong Institute of Finance, Guangzhou College of South China University of Technology, Guangdong Medical University, Guangdong Ocean University, Guangzhou Institute of Physical Education and Guangzhou Academy of Fine Arts. 1800 questionnaires were distributed and 1628 valid questionnaires were collected, with an effective rate of $90.4 \%$. The average age was (20.8 \pm 3.9$)$ years. There are 900 boys and 728 girls, 689 urban students and 939 rural students, 422 freshmen, 389 sophomores, 407 juniors, 317 seniors and 93 seniors, 663 engineering students, 357 science students, 391 liberal arts students, 135 art students and 82 sports students.

\subsection{Tools \\ 2.2.1. Resilience Scale for Chinese Adolescent (RSCA)}

Self-evaluation scale complied by Hu and Gan (2008). There are 27 items, divided into five factors: goal focus (GF), emotion control (EC), positive cognition (PC), family support (FS) and interpersonal assistance (IA). The first three factors belong to the domain of personal strengh (PS), and the latter two are in the domain of support strengh (SS). Likert 5-point scoring method is used to score from 1 to 5 points corresponding to "completely inconsistent" to "completely consistent". The higher the total score, the higher the degree of resilience.

\subsubsection{Academic Burnout Scale for Chinese Undergraduates (ABSCU)}

It is complied by Lian, Yang, and Wu (2005) according to Maslash Job Burnout scale, which is a self-evaluation scale and used to evaluate the academic burnout of college students. There are 20 items, divided into three dimensions: low mood (LD), improper behavior (IB) and low sense of achievement (LSA). Likert 5-point scoring method is used to score from 1 to 5 points corresponding to "completely inconsistent" to "completely consistent". The higher the score, the more obvious the tendency in the item, dimension or learning burnout.

\subsubsection{Self-Compiled Personal Information Questionnaire}

It includes 4 items, namely, gender, grade, origin, college.

\subsection{Collection and Arrangement of Aata}

Before the investigation, the researchers who participated in the survey were trained uniformly, and the investigation process and evaluation standard were unified. The consistency test (kappa $=0.81-0.90)$ meet the test requirements. The questionnaires with scores of more than $50 \%$ of the items missing were eliminated. The missing values of the valid questionnaire were estimated and filled with the average. Two researchers independently input the same data using Epidata3.0 software and conduct a unified logic check to ensure the accuracy of the data.

\subsection{Statistical Methods}

Data are exported from Epidata3.0 to SPSS 20.0 for statistical analysis. First, the original score of the total scale,each dimension and domainare calculated. The second step is to get the average score and standard deviation 
of the total scale, each dimension and domain. In the third step, the floor and ceiling effect are evaluated, and then, Cronbach's $\alpha$ coefficient and Split half reliability are calculated. Finally, convergent validity, discriminant validity and principal component factor analysis are conducted.

Table-1. Scoring Method of RSCA.

\begin{tabular}{lccc}
\hline Dimension & Number of items & Item distribution & Range of original scores \\
\hline Goal focused (GF) & 5 & $3,4,11,20,24$ & $0-25$ \\
Emotion control (EC) & 6 & $1,2,5,21,23,27$ & $0-30$ \\
Positive cognition (PC) & 4 & $10,13,14,25$ & $0-20$ \\
Family support (FS) & 6 & $8,15,16,17,19,22$ & $0-30$ \\
Interpersonal assistance & 6 & $6,7,9,12,18,26$ & $0-30$ \\
(IA) & & & \\
\hline
\end{tabular}

\section{RESULTS}

\subsection{Score Distribution of RSCA}

The ceiling / floor effect is one of the psychological test effects, which refers to the phenomenon that when a test is too simple / complex, the scores of most subjects are close to or reach the upper / lower limit of scores, which makes the evaluation and prediction performance of the test decrease (Zhang \& Xu, 2012). Table 2 shows that the total score of RSCA and the scores of 2 domains and 5 dimensions all tend to be normal distribution, without any celling or floor effect.

Table-2.Descriptive Analysis of RSCA ( $\mathrm{n}=1628)$.

\begin{tabular}{|c|c|c|c|c|c|c|c|c|}
\hline Dimension/domain & $\mathbf{X} \pm \mathbf{s}$ & Min & Max & P25 & P50 & P75 & Floor $[\mathbf{n}(\%)]$ & Celling $[\mathbf{n}(\%)]$ \\
\hline Goal focused (GF) & $17.4 \pm 3.4$ & 5.0 & 25.0 & 15.0 & 18.0 & 20.0 & $4(0.2)$ & $34(2.1)$ \\
\hline Emotion control (EC) & $18.9 \pm 3.9$ & 6.0 & 30.0 & 16.0 & 19.0 & 21.0 & $2(0.1)$ & $\mathrm{O}(\mathrm{O})$ \\
\hline Positive cognition (PC) & $14.9 \pm 3.1$ & 4.0 & 20.0 & 13.0 & 15.0 & 17.0 & $6(0.4)$ & $108(6.6)$ \\
\hline Family support (FS) & $21.4 \pm 3.8$ & 6.0 & 30.0 & 19.0 & 21.0 & 24.0 & $2(0.1)$ & $12(0.7)$ \\
\hline $\begin{array}{l}\text { Interpersonal assistance } \\
\text { (IA) }\end{array}$ & $19.8 \pm 4.3$ & 6.0 & 30.0 & 17.0 & 20.0 & 23.0 & $5(0.3)$ & $17(1.0)$ \\
\hline Personal strength (PS) & $51.1 \pm 7.4$ & 23.0 & 75.0 & 46.0 & 51.0 & 56.0 & $2(0.1)$ & $\mathrm{O}(\mathrm{O})$ \\
\hline Support strength (SS) & $41.1 \pm 6.6$ & 12.0 & 68.0 & 36.0 & 41.0 & 46.0 & $\mathrm{O}(0)$ & $\mathrm{O}(\mathrm{O})$ \\
\hline Total score of RSCA & $92.3 \pm 12.1$ & 35.0 & 135.0 & 83.0 & 91.0 & 100.0 & $\mathrm{O}(\mathrm{O})$ & $\mathrm{O}(\mathrm{O})$ \\
\hline
\end{tabular}

\subsection{Reliability Analysis of RSCA \\ 3.2.1. Split Half Reliability}

27 items of RSCA are divided into two parts with 13 items and 14 items, respectively, and the correlation coefficient of these two parts is $\mathrm{r}=0.68(\mathrm{P}<0.01)$. According to Sperman Brown formula, the split half reliability of the total scale is 0.81 . The correlation coefficients of two halves of five factors are $0.65,0.50,0.75,0.60$ and 0.54 , and the split half reliability are $0.82,0.66,0.88,0.76$ and 0.71 , respectively. The correlation coefficients of two parts of personal and support strength are 0.75 and 0.68 , respectively, and the split half reliability are 0.89 and 0.83 , respectively.

\subsubsection{Internal Consistency Reliability}

Cronbach's $\alpha$ coefficient is used to measure the internal consistency reliability of each factor. Generally speaking, when Cronbach's $\alpha$ coefficient is greater than 0.7, the internal consistency reliability is better. It can be seen from Table 3 that Cronbach's $\alpha$ coefficient of the total table is 0.86, and Cronbach's $\alpha$ coefficients of five factors are 0.81, 0.76, 0.74, 0.72 and 0.77, respectively. Cronbach's $\alpha$ coefficients of personal and support strength are 0.86 and 0.81 , 
respectively. There is a moderate to low correlation between factors, a moderate to high correlation between each factor and its domain, and a moderate to high correlation between each domain and the total score of RSCA.

\begin{tabular}{|c|c|c|c|c|c|c|c|c|c|}
\hline Dimension/domain & Cronbach's $\alpha$ & 1 & 2 & 3 & 4 & 5 & 6 & 7 & 8 \\
\hline 1. GF & 0.81 & & & & & & & & \\
\hline 2. EC & 0.76 & $0.16^{* * *}$ & & & & & & & \\
\hline 3. $\mathrm{PC}$ & 0.74 & $0.58^{* *}$ & $0.08^{* *}$ & & & & & & \\
\hline 4. FS & 0.72 & $0.37^{* *}$ & $0.14^{* *}$ & $0.40^{* *}$ & & & & & \\
\hline 5. IA & 0.77 & $0.26^{* *}$ & $0.30^{* *}$ & $0.23^{* *}$ & $0.33^{* *}$ & & & & \\
\hline 6. PS & 0.84 & $0.78^{* *}$ & $0.64^{* *}$ & $0.72^{* * *}$ & $\begin{array}{c}0.41 \\
* * *\end{array}$ & $0.37^{* *}$ & & & \\
\hline 7. SS & 0.81 & $0.38^{* *}$ & $0.27^{* *}$ & $0.38^{* *}$ & $0.79^{* *}$ & $0.84^{* *}$ & $0.48^{* *}$ & & \\
\hline 8.total score of RSCA & 0.86 & $0.69^{\text {*** }}$ & $0.54^{* * *}$ & $0.65^{* * *}$ & $0.68^{* * *}$ & $0.69^{* * *}$ & $0.88^{* * *}$ & $0.84^{* * *}$ & \\
\hline
\end{tabular}

\subsection{Validity Analysis of RSCA \\ 3.3.1. Content Validity}

The correlation coefficient $(R)$ between each item and its dimension is used to represent the convergent validity. Generally, when $R \geq 0.4$, it can be considered that the convergent validity is better. Discriminant validity is expressed by the correlation coefficient between the item and other dimensions. If these correlation coefficients are lower than the correlation coefficient between the item and its dimension, the discriminant validity is better. The results show that the correlation coefficient between each item and its dimension is $>0.4$, which is higher than those correlation coefficients between the same item with other dimensions. The success rates of calibration of convergent and discriminant validity of each factor are $100 \%$, the success rates of calibration of convergent validity of two domains are both above $86.7 \%$, and the success rates of calibration of discriminant validity of 2 domains are $100 \%$. See Table 4.

Table-4.Convergentvalidity and discriminant validity of RSCA.

\begin{tabular}{|c|c|c|c|c|c|c|c|}
\hline \multirow{2}{*}{$\begin{array}{c}\text { Dimension/ } \\
\text { Domain }\end{array}$} & \multirow{2}{*}{$\begin{array}{l}\text { number } \\
\text { of items }\end{array}$} & \multicolumn{3}{|c|}{ Convergent Vality } & \multicolumn{3}{|c|}{ Discriminant Vality } \\
\hline & & Range of $R$ & Success & $\begin{array}{c}\text { Success rate } \\
(\%)\end{array}$ & Range of $R$ & Success & $\begin{array}{l}\text { Success } \\
\text { rate }(\%)\end{array}$ \\
\hline GF & 5 & $0.645-0.728$ & $5 / 5$ & 100 & 003-0.466 & $20 / 20$ & 100 \\
\hline $\mathrm{EC}$ & 6 & $0.480-0.721$ & $6 / 6$ & 100 & $0.010-0.420$ & $24 / 24$ & 100 \\
\hline $\mathrm{PC}$ & 4 & $0.744-0.782$ & $4 / 4$ & 100 & $0.009-0.507$ & $16 / 16$ & 100 \\
\hline FS & 6 & $0.445-0.713$ & $6 / 6$ & 100 & $0.030-0.412$ & $24 / 24$ & 100 \\
\hline IA & 6 & $0.552-0.670$ & $6 / 6$ & 100 & $0.006-0.304$ & $24 / 24$ & 100 \\
\hline PS & 15 & $0.279-0.621$ & $13 / 15$ & 86.7 & $0.077-0.349$ & $15 / 15$ & 100 \\
\hline SS & 12 & $0.278-0.600$ & $11 / 12$ & 91.7 & $0.063-0.374$ & $12 / 12$ & 100 \\
\hline
\end{tabular}

\subsubsection{Construct Validity}

As KMO value is 0.979 , Bartlett's spherical test value is $11344.65(\mathrm{df}=351), P<0.001$, the data is suitable for factor analysis. According to the eigenvalue greater than 1.0, 5 principal components are extracted, with a cumulative contribution rate of $48.97 \%$, which can basically reflect the theoretical conception of the original scale (the load of each factor and eigenvalue are shown in Table 5, and the gravel diagram of each factor and eigenvalue are shown in Figure 1. Further principal component analysis of five factors shows that KMO value is 0.68, Bartlett's spherical test value is $1409.80(\mathrm{df}=10, P<0.001)$, so the data is also suitable for factor analysis. Two principal components are extracted according to the eigenvalue greater than 1.0, with a cumulative contribution rate of $65.23 \%$ (see Table 6 for the load of each factor and eigenvalue, and see Figure 2 for the gravel diagram of each factor and eigenvalue). 
Table-5. Principal Component Analysis of 27 Items of RSCA and Load of Each Factor (>0.5).

\begin{tabular}{|c|c|c|c|c|c|c|c|c|c|}
\hline \multicolumn{2}{|c|}{$\begin{array}{l}1^{\text {st }} \text { Principal } \\
\text { component }\end{array}$} & \multicolumn{2}{|c|}{$\begin{array}{c}2^{\text {nd }} \text { Principal } \\
\text { component }\end{array}$} & \multicolumn{2}{|c|}{$\begin{array}{c}3^{\text {rd }} \text { Principal } \\
\text { component }\end{array}$} & \multicolumn{2}{|c|}{$\begin{array}{l}4^{\text {th }} \text { Principal } \\
\text { component }\end{array}$} & \multicolumn{2}{|c|}{$\begin{array}{c}5^{\text {th }} \text { Principal } \\
\text { component }\end{array}$} \\
\hline item & $\begin{array}{c}\text { Factor } \\
\text { load }\end{array}$ & item & $\begin{array}{c}\text { Factor } \\
\text { load }\end{array}$ & item & $\begin{array}{l}\text { Factor } \\
\text { load }\end{array}$ & item & $\begin{array}{c}\text { Factor } \\
\text { load }\end{array}$ & item & $\begin{array}{l}\text { Factor } \\
\text { load }\end{array}$ \\
\hline 3 & 0.534 & 1 & 0.736 & 10 & 0.632 & 8 & 0.520 & 6 & 0.533 \\
\hline 4 & 0.571 & 2 & 0.784 & 13 & 0.620 & 15 & 0.709 & 7 & 0.502 \\
\hline 11 & 0.581 & 5 & 0.644 & 14 & 0.634 & 16 & 0.739 & 9 & 0.565 \\
\hline 20 & 0.592 & 21 & 0.603 & 25 & 0.720 & 17 & 0.720 & 12 & 0.646 \\
\hline 24 & 0.587 & 23 & 0.562 & & & 19 & 0.517 & 18 & 0.604 \\
\hline & & 27 & 0.586 & & & 22 & 0.503 & 26 & 0.670 \\
\hline
\end{tabular}

Table-6. Principal Component Analysis of Five Factors of RSCA and Load of Each Factor (>0.5).

\begin{tabular}{ccccc|}
\hline \multicolumn{2}{c}{$\mathbf{1}^{\text {st}}$ Principal component } & \multicolumn{2}{c|}{ 2 $^{\text {nd Principal component }}$} \\
Factor & Domain load & Factor & Domain load \\
\hline GF & 0.814 & FS & 0.859 \\
\hline EC & 0.863 & IA & 0.710 \\
\hline PC & 0.660 & & \\
\hline
\end{tabular}

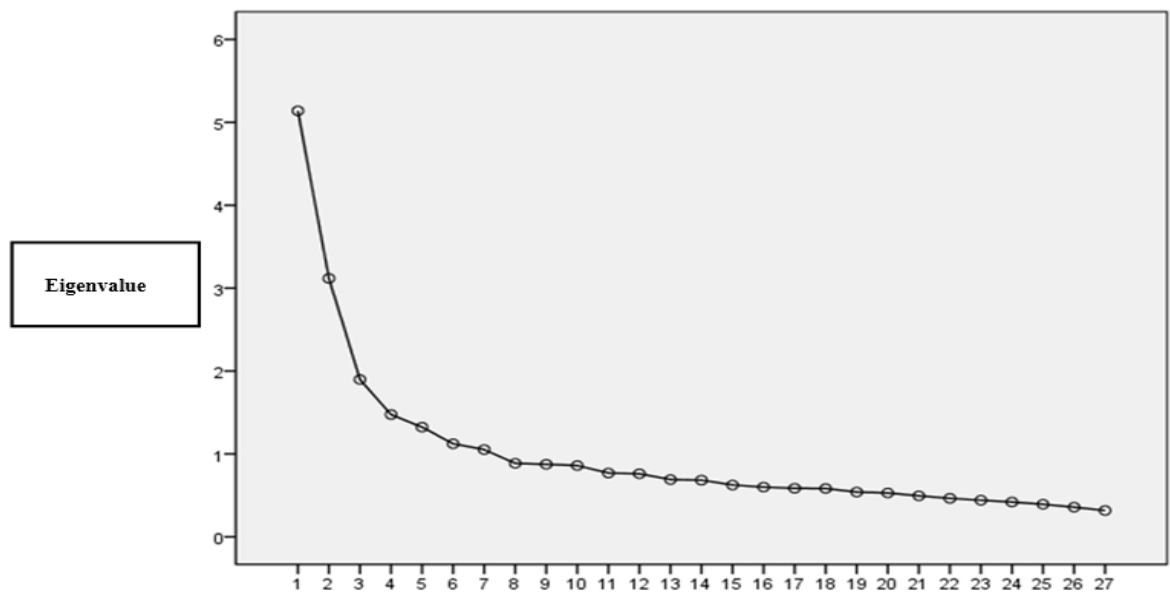

number of components

Figure-1. Results of principal component analysis for 27 items of RSCA.

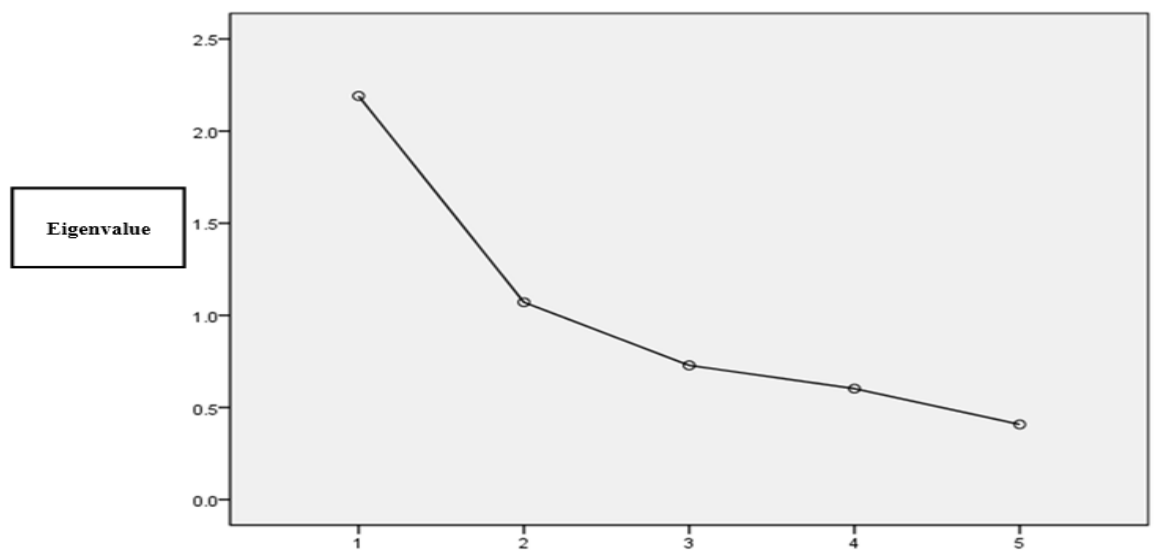

Figure-2. Results of principal component analysis for 5 factors of RSCA

\subsubsection{Criterion Validity}

Taking the total score of RSCA as the independent variable and the total score of ABSCU as the dependent variable, a multiple stepwise linear regression is carried out within the $95 \%$ confidence interval. 
From Table 5, it can be found that the total score of RSCA entered the regression equation, and the standardized coefficient (correlation coefficient) is $-0.398, \mathrm{r}^{2}$ adj $=0.158$. In other words, the total score of RSCA can significantly predict the total score of ABSCU.

Table-7. Linear Regression Analysis of RSCA on the Total Score of ABSCU.

\begin{tabular}{ccccccccc}
\hline Dependent Variable & \multicolumn{2}{c}{ Regression coefficient } & & $\boldsymbol{\beta}$ & $\boldsymbol{t}_{\text {value }}$ & $\boldsymbol{P}_{\text {value }}$ & $\boldsymbol{R}^{\boldsymbol{e}}$ & $\boldsymbol{R}_{\text {adj }}{ }^{2}$ \\
\hline Total score of ABSCU & Total score of RSCA & B & SE & -0.398 & -17.489 & $<0.001$ & 0.158 & 0.158 \\
\hline & & - & 0.019 & & & & \\
& & 0.329 & & & & & \\
\hline
\end{tabular}

\section{DICUSSION}

This study finds that the psychometric performance of RSCA applied to college students in Guangdong Province is good, which is consistent with the results of previous literature (Chen et al., 2020; Hu \& Gan, 2008; Xu \& Yang, 2019), suggesting that RSCA is suitable for college students in Guangdong Province. First, the results of internal consistency reliability, split half reliability, construct validity (convergent validity, discriminant validity, principal component analysis) and ceiling/floor effect show that RSCA has good psychometric performance. The internal consistency reliability of the total scale, 2 domains and 5 factors of RSCA are all above 0.72 ; the split half reliability of the total scale is 0.81 , and the split half reliability of 2 domains and 5 factors are $0.66-0.88$, which is consistent with the results of previous studies (Chen et al., 2020; Hu \& Gan, 2008; Xu \& Yang, 2019), suggesting that the split half reliability and internal consistency reliability of RSCA applied to college students in Guangdong Province are good. Sceond, we find that each item of RSCA is highly correlated with its dimension, and each dimension is also highly correlated with the total scale, which is consistent with the results of previous study (Hu \& Gan, 2008). Principal component analysis is used to analyze the scores of 27 items of RSCA, and 5 principal components are extracted, with a cumulative contribution rate of $48.97 \%$, Further principal component analysis of five factors and 2 principal components are extracted, with a cumulative contribution rate of $65.23 \%$, which basically meet the theoretical conception of RSCA and indicate that RSCA has good construct validity. Third, we find that the correlation coefficients ofeach item of RSCA with the factorwhere the item is located is $\geq 0.4$, and the correlation coefficientsof each itemwith other factors are less than the correlation coefficients between the same item and the factor where the item is located. The calibration success rates of convergent validity and discriminant validity of each factor are $100 \%$, and the calibration success rates of convergent validity and discriminant validity of the two domains are above $86.7 \%$. It is consistent with the results of previous literature (Hu \& Gan, 2008), suggesting that RSCA has good convergent validity and discriminant validity. On the other hand, the regression analysis shows that the total score of RSCA can significantly predict the total score of ABSCU, indicating that the criterion validity of RSCA is good. Final, the resilience of this group is generally good. The total score, five factors and two domains of the scale tend to be normal distribution, without any ceiling or floor effect. It is suggested that the item selection of RSCA is appropriate (the behavior sampling is typical), the scale development is reasonable, and the sensitivityis good.

\section{REFERENCES}

American Psychology Association. (2011). The road to resilience: What is resilience [OL/EB]? Retrieved from: http://www.apahelpcenter.org/ featuredtopics/ feature. php ? id=6\&ch=2, 2004/12/ 30 .

Bulut, S., Doğan, U., \& Altundağ, Y. (2013). Adolescent psychological resilience scale: Validity and reliability study. Contemporary Psychology, 16(1), 21-32. 
Cao, L. (2020). Research on the relationship between resilience and quality of life, education environment perception of medical students. Chinese Journal of Multimedia and Network Teaching, 26(2), 212-213.

Chen, J., Li, H., \& Yang, Y. (2019). The influence of family resilience on resilience of cancer patients: Chain mediating effect of understanding social support and sense of life significance Chinese Journal of Clinical Psychology, 27(6), 1205-1209.

Chen, J., Li, H., \& Yang, Y. (2020). The mediating role of College Students' resilience between mobile phone addiction and mental health. Modern Preventive Medicine, 47(1), 93-96.

Chen, J., Zhao, W., \& Wang, P. (2020). The mediating role of academic self-efficacy in the relationship between resilience and academic delayed satisfaction of college students. Medical Education Research and Practice, 28(2), 285-290.

Cohen, J. (1992). Statistical power analysis. Curr Direct Psychol Science, 1(3), 98-101.

Gao, J., \& Sui, G. (2018). Research on the influence of College Students' resilience and decision-making strategies on career decision-making difficulties. Journal of Yangzhou University, 22(3), 62-67.

Heppner, P. P., Heppner, M. J., Lee, D.-G., Wang, Y.-W., Park, H.-J., \& Wang, L.-F. (2006). Development and validation of a collectivist coping styles inventory. Journal of Counseling Psychology, 53(1), 107-125.

Hou, Y., Hu, P., \& Wang, Y. (2017). The influence of learning burnout on medical students' learning burnout: The mediating role of coping style. Progress in Psychology, 7(7), 845-851.

Hu, Y., \& Gan, Y. (2008). Development and validation of Adolescent Resilience Scale. Acta Psychologica Sinica, 5(8), 902-912.

Li, J., Yin, L., \& Liu, X. (2014). The influence of core self-evaluation on Learning Burnout of Sports Majors: The mediating role of coping style. Sports Science and Technology, 36(3), 94-96.

Li, Q., Niu, y., \& Ma, H. (2020). The relationship between campus cold violence and coping style of college students: The mediating role of resilience. Ergonomics, 26(2), 58-63.

Lian, R., Yang, L., \& Wu, L. (2005). The relationship between college students' professional commitment and learning burnout and the development of the scale. Acta Psychologica Sinica, 37(5), 632-636.

Lin, S., Luo, M., \& Wang, D. (2019). Research status of resilience measurement tools. Chinese PLA Journal of Nursing, 36(4), 5457.

Liu, D., \& Zhouyuqiu, L. (2019). Social support and schizophrenia disability: Chain mediating effect of self-esteem and resilience. Chinese Journal of Clinical Psychology, 27(1), 78-83.

Pan, Y., \& Yang, G. (2013). Review of foreign research on resilience. Journal of Guizhou Normal University, 33(3), 7-12.

Saide, Ö. (2019). Validity and reliability study of social emotional well-being and psychological resilience scale for preschool children (PERIK). OPUS International Journal of Society Researches, 17(5), 756-786.

Sang, L., Chen, G., \& Zhu, J. (2016). The relationship between social support and learning adaptation of college students: The mediating role of resilience. Chinese Journal of Health Psychology, 24(2), 248-252.

Xu, M., \& Yang, X. (2019). The influence of big five personality on middle school students' suicidal ideation: The mediating effect and moderating effect of resilience. Educational Measurement and Evaluation, 3(1), 57-64.

Yu, X., Yin, M., Zhao, Y., \& Xin, S. (2020). A cross-sectional historical study on the changes of learning burnout of Chinese college students. Psychological Technology and Application, 8(2), 74-83.

Zhang, H., \& Xu, J. (2012). Modern psychology and educational statistics. Beijing: Beijing Normal University Publishing Group. 